\title{
Erratum to: Hazard levels of warning signal words modulate the inhibition of return effect: evidence from the event-related potential P300
}

\author{
Qian Shang ${ }^{1}$ Yujing Huang ${ }^{2}$ Qingguo $\mathrm{Ma}^{3}$
}

Published online: 26 March 2016

(C) Springer-Verlag Berlin Heidelberg 2016

Erratum to: Exp Brain Res (2015) 233:2645-2653

DOI 10.1007/s00221-015-4335-4

The institutional affiliation of the first author, Qian Shang, was incorrectly rendered in the original publication of this paper. It was misspelled.

His affiliation should read as follows:

1 Management School, Hangzhou Dianzi University, Hangzhou, People's Republic of China.

The online version of the original article can be found under doi:10.1007/s00221-015-4335-4.

Qingguo Ma

maqingguo3669@zju.edu.cn

1 Management School, Hangzhou Dianzi University,

Hangzhou, People's Republic of China

2 School of Economics and Management, Zhejiang Sci-tech

University, Hangzhou, People's Republic of China

3 Neuromanagement Laboratory, School of Management,

Zhejiang University, 38\# Zheda Road, Hangzhou 310027,

People's Republic of China 